\title{
A COMMUTATIVITY CRITERION FOR $\delta$-PRIME RINGS
}

\author{
KAMIL KULAR
}

Received 07 April, 2017

\begin{abstract}
We prove that under some natural assumptions, a $\delta$-prime ring is commutative whenever so is the range of the derivation $\delta$. We also provide a few useful observations on $\delta$-prime rings, related to the main theorem.
\end{abstract}

2010 Mathematics Subject Classification: 16W25; 16U99

Keywords: associative ring, derivation, $\delta$-ideal, $\delta$-prime ring, commutativity

\section{Preliminaries AND introduction}

Throughout the present note, $R$ is an associative ring, possibly without identity, and $\delta: R \longrightarrow R$ is a derivation. The latter means that the map $\delta$ is additive and satisfies the Leibniz rule

$$
\forall x, y \in R: \delta(x y)=\delta(x) y+x \delta(y) .
$$

We will denote by $\mathbb{N}$ the set of non-negative integers (and by $\mathbb{Z}$ the set of all integers). We define

$$
\delta^{n}= \begin{cases}\mathrm{id}_{R}, & \text { if } n=0, \\ \underbrace{\delta \circ \ldots \circ \delta}_{n}, & \text { if } n \in \mathbb{N} \backslash\{0\} .\end{cases}
$$

The commutator of elements $x, y \in R$ is defined by $[x, y]=x y-y x$. Let us recall that a set $E \subseteq R$ is said to be $\delta$-stable, if $\delta(E) \subseteq E$. A left, right, or two-sided ideal $I$ of the ring $R$ is called a $\delta$-ideal, if it is a $\delta$-stable set. Observe that the intersection of an arbitrary family of two-sided $\delta$-ideals of the ring $R$ is again a two-sided $\delta$-ideal of $R$. This fact enables us to consider two-sided $\delta$-ideals generated by subsets of $R$. In the latter two sentences, the word "two-sided" can obviously be replaced by "left" or "right". The ring $R$ is said to be 2-torsion free, if

$$
\forall x \in R: 2 x=0 \Longrightarrow x=0 .
$$

The centralizer, the left annihilator and the right annihilator of a set $E \subseteq R$ are defined by

$$
\begin{aligned}
& \mathrm{C}_{R}(E)=\{a \in R: a x=x a \text { for all } x \in E\}, \\
& \operatorname{ann}_{\ell}(E)=\{a \in R: a x=0 \text { for all } x \in E\},
\end{aligned}
$$

(C) 2017 Miskolc University Press 


$$
\operatorname{ann}_{r}(E)=\{a \in R: x a=0 \text { for all } x \in E\},
$$

respectively. It is easy to see that $\operatorname{ann}_{\ell}(E)$ is a left ideal of the $\operatorname{ring} R$ and $\operatorname{ann}_{r}(E)$ is a right ideal of $R$. Moreover, if $E$ is $\delta$-stable, then both annihilators are $\delta$-ideals. We denote by $\mathrm{Z}(R)$ the center of $R$, i.e.,

$$
\mathrm{Z}(R)=\mathrm{C}_{R}(R)=\{a \in R: a x=x a \text { for all } x \in R\} .
$$

The present note is concerned with $\delta$-prime rings. Let us recall that the ring $R$ is said to be $\delta$-prime, if it is nonzero and the product of any two its nonzero two-sided $\delta$-ideals is again nonzero. Notice that $R$ is prime if and only if it is 0 -prime (i.e., $\delta$-prime with $\delta$ being the zero derivation). A typical example of a $\delta$-prime ring which is not even semiprime can be found, for instance, in [8]. The theorem below provides among other things a useful characterization of $\delta$-prime rings in terms of annihilators. For the proof of the theorem we refer to [9].

Theorem 1. Suppose that $R$ is a nonzero ring. The following conditions are equivalent:

(1) $R$ is $\delta$-prime,

(2) for any $a, b \in R$, if $\forall n \in \mathbb{N}: a R \delta^{n}(b)=\{0\}$, then $a=0$ or $b=0$,

(3) for any $a, b \in R$, if $\forall n \in \mathbb{N}: \delta^{n}(a) R b=\{0\}$, then $a=0$ or $b=0$,

(4) for any $a, b \in R$, if the product of the two-sided $\delta$-ideal of $R$ generated by $a$ and the two-sided $\delta$-ideal of $R$ generated by $b$ is equal to $\{0\}$, then $a=0$ or $b=0$,

(5) the product of any two nonzero left $\delta$-ideals of $R$ is again nonzero,

(6) the product of any two nonzero right $\delta$-ideals of $R$ is again nonzero,

(7) for an arbitrary nonzero left $\delta$-ideal $I$ of the ring $R$ we have $\operatorname{ann}_{\ell}(I)=\{0\}$,

(8) for an arbitrary nonzero right $\delta$-ideal $J$ of the ring $R$ we have $\operatorname{ann}_{r}(J)=\{0\}$.

Some further information about $\delta$-prime rings can be found in [2]. For an exhaustive historical account on derivations in abstract algebra we refer to [3].

Derivations on rings such that the elements in the range commute have been studied extensively over the years. The study of such derivations on prime rings of characteristic different from 2 was initiated by Herstein in [6]. The results of Herstein were then generalized in Daif's paper [4] to semiprime rings. At the present time, a considerable number of various modifications and extensions of Herstein and Daif's original results are known (see, for instance, [1,5,11]; we also refer to [10] for a general survey of commutativity conditions for rings). Most of these modifications and extensions require the underlying ring to be prime or at least semiprime. The present note generalizes some of the results in $[6,7]$ to $\delta$-prime rings. Our main purpose is to prove that if the ring $R$ is $\delta$-prime, then under certain natural assumptions the commutativity of $\delta(R)$ implies the commutativity of $R$. 


\section{THE RANGE OF A DERIVATION}

Let us begin with a slightly enhanced version of [7, Theorem 1].

Proposition 1. Suppose that $\delta^{3} \neq 0$. Then the subring $S$ of the ring $R$ generated by $\delta(R)$ contains a nonzero two-sided $\delta$-ideal of $R$.

Proof. Let $I$ be the two-sided $\delta$-ideal of $R$ generated by the set $\delta^{3}(R)$. Obviously, $I \neq\{0\}$. We will show that $I \subseteq S$. Observe first that $I$ coincides with the totality of finite sums of elements of the set $A \cup B \cup C \cup \delta^{3}(R)$, where $A=\left\{a \delta^{3}(x): a, x \in R\right\}$, $B=\left\{\delta^{3}(x) b: b, x \in R\right\}$ and $C=\left\{a \delta^{3}(x) b: a, b, x \in R\right\}$. Consequently, it is enough to prove that $A \cup B \cup C \cup \delta^{3}(R) \subseteq S$. Notice that $\delta^{3}(R) \subseteq \delta(R)$. Now, pick any $r, y \in R$. Then

$$
\delta(r \delta(y))=\delta(r) \delta(y)+r \delta^{2}(y) .
$$

Since $\delta(r \delta(y))$ and $\delta(r) \delta(y)$ belong to the subring $S$, we therefore get $r \delta^{2}(y) \in S$. In the same way one can prove that $\delta^{2}(y) r \in S$. Hence

$$
A=\left\{a \delta^{2}(\delta(x)): a, x \in R\right\} \subseteq S,
$$

and analogously $B \subseteq S$. It remains to show that $C \subseteq S$. For arbitrary $a, b, x \in R$ we have

$$
\delta\left(a \delta^{2}(x) b\right)=\delta(a) \delta^{2}(x) b+a \delta^{3}(x) b+a \delta^{2}(x) \delta(b) .
$$

Since $a \delta^{2}(x)$ and $\delta^{2}(x) b$ are elements of $S$, the above equality implies that $a \delta^{3}(x) b \in$ $S$. The desired inclusion follows.

The slight enhancement thus consists in replacing a "usual" ideal of the ring $R$ with a $\delta$-ideal of $R$. However, the proof which we have presented is in fact Herstein's original proof, with a few technical modifications. (We include this proof for the sake of completness). This is because the "usual" two-sided ideal of $R$ generated by $\delta^{3}(R)$ is $\delta$-stable.

\section{AuXiLiary RESUltS}

We now start with a simple lemma concerning two derivations.

Lemma 1. Let $I$ be a nonzero left or right $\delta$-ideal of the ring $R$. Moreover, let $d: R \longrightarrow R$ be a derivation. Suppose that $R$ is $\delta$-prime and $d$ vanishes on I. Then $d=0$.

Proof. Assume that $I$ is a left $\delta$-ideal. Pick any $a \in I$ and any $x \in R$. Since $R I \subseteq I$, the Leibniz rule yields $0=d(x a)=d(x) a$. Consequently, $d(R) \subseteq \operatorname{ann}_{\ell}(I)$. It follows therefore from the $\delta$-primeness that $d(R)=\{0\}$. If $I$ is a right $\delta$-ideal, we can proceed analogously.

Recall that for any fixed $a \in R$, the map $\partial_{a}: R \ni x \longmapsto[a, x] \in R$ is a derivation. This derivation is referred to as the inner derivation of the ring $R$ induced by $a$. 
Lemma 2. Let I be as in the previous lemma. Suppose, again, that the ring $R$ is $\delta$-prime. Then $R$ is commutative whenever so is $I$.

Proof. Assume that the $\delta$-ideal $I$ is commutative. Pick an arbitrary $a \in I$. By the commutativity of $I$, the inner derivation $\partial_{a}: R \longrightarrow R$ vanishes on $I$. The previous lemma therefore implies that $\partial_{a}(x)=[a, x]=0$ for any $x \in R$. Thus we have $I \subseteq$ $\mathrm{Z}(R)$. Now, pick an arbitrary $b \in R$. Since $I \subseteq \mathrm{Z}(R)$, the inner derivation $\partial_{b}$ vanishes on $I$. Consequently, $[b, x]=0$ for all $x \in R$. The commutativity of $R$ follows.

The next lemma is a generalized version of [6, Lemma 1.1.7].

Lemma 3. Let $u \in R$ and $k \in \mathbb{N}$. Then the set

$$
A_{\delta}^{k}(u)=\operatorname{ann}_{\ell}\left(\left\{\left[\delta^{m}(u), x\right]: x \in R, m \in \mathbb{N}, m \geqslant k\right\}\right)
$$

is a two-sided $\delta$-ideal of the ring $R$. Moreover, if $R$ is $\delta$-prime and $A_{\delta}^{0}(u) \neq\{0\}$, then $u \in \mathrm{Z}(R)$.

Proof. Obviously, $A_{\delta}^{k}(u)$ is a left ideal of $R$. Pick arbitrary elements $a \in A_{\delta}^{k}(u)$ and $x, y \in R$. Let $m \in \mathbb{N}$ be such that $m \geqslant k$. Then we have

$$
0=a\left[\delta^{m}(u), y x\right]=a\left[\delta^{m}(u), y\right] x+a y\left[\delta^{m}(u), x\right]=a y\left[\delta^{m}(u), x\right] .
$$

This yields that $a y \in A_{\delta}^{k}(u)$ for any $a \in A_{\delta}^{k}(u)$ and any $y \in R$. Hence $A_{\delta}^{k}(u)$ is a right ideal of $R$. We will show that $A_{\delta}^{k}(u)$ is $\delta$-stable. If $a \in A_{\delta}^{k}(u), x \in R, m \in \mathbb{N}$ and $m \geqslant k$, then

$$
\begin{aligned}
0 & =\delta\left(a\left[\delta^{m}(u), x\right]\right)= \\
& =\delta(a)\left[\delta^{m}(u), x\right]+a\left[\delta^{m+1}(u), x\right]+a\left[\delta^{m}(u), \delta(x)\right]= \\
& =\delta(a)\left[\delta^{m}(u), x\right] .
\end{aligned}
$$

Consequently, $\delta(a) \in A_{\delta}^{k}(u)$ for an arbitrary $a \in A_{\delta}^{k}(u)$.

Let us turn to the "moreover" part. Suppose that $R$ is a $\delta$-prime ring and $A_{\delta}^{0}(u) \neq$ $\{0\}$. Since $A_{\delta}^{0}(u)$ is a two-sided $\delta$-ideal of $R$, it follows that $\operatorname{ann}_{r}\left(A_{\delta}^{0}(u)\right)=\{0\}$. But

$$
\{[u, x]: x \in R\}=\left\{\left[\delta^{0}(u), x\right]: x \in R\right\} \subseteq \operatorname{ann}_{r}\left(A_{\delta}^{0}(u)\right) .
$$

Therefore $[u, x]=0$ for all $x \in R$. This means that $u \in \mathrm{Z}(R)$.

In [6] (proof of Lemma 1.1.9), Herstein showed that if $R$ is a semiprime ring of characteristic different from 2 and $\delta^{2}=0$, then $\delta=0$. The first part of the proposition below is a modification of the result: we impose a weaker condition on $\delta$ and assume that $R$ is $\delta$-prime. Before starting the proposition, notice that $R^{2}$ is a two-sided $\delta$ ideal of the ring $R$.

Proposition 2. Let $R$ be a 2-torsion free $\delta$-prime ring.

(i) If $\delta^{2}\left(R^{2}\right)=\{0\}$, then $\delta=0$.

(ii) If $\delta \neq 0$, then $\mathrm{C}_{R}(\delta(R))=\mathrm{Z}(R)$. 
Proof. Suppose that $\delta^{2}$ vanishes on $R^{2}$. Define $K \subseteq R$ to be the totality of finite sums of elements of the form $a \delta(x) b$, where $a, b \in R$ and $x \in R^{2}$. Then $K$ is a twosided $\delta$-ideal of the ring $R$. Now, pick arbitrary $r, s \in R^{2}$. Since $R$ is 2-torsion free and

$$
0=\delta^{2}(r s)=\delta^{2}(r) s+2 \delta(r) \delta(s)+r \delta^{2}(s)=2 \delta(r) \delta(s),
$$

we get $\delta(r) \delta(s)=0$. Consequently, if $x, y, z \in R^{2}$, then

$$
0=\delta(x) \delta(z y)=\delta(x) \delta(z) y+\delta(x) z \delta(y)=\delta(x) z \delta(y) .
$$

It follows that $a \delta(x) b f \delta(y) g=0$ for any $a, b, f, g \in R$ and any $x, y \in R^{2}$. This yields $K^{2}=\{0\}$. In view of the $\delta$-primeness of $R$, we have therefore $K=\{0\}$. Again let $x \in R^{2}$. Since

$$
\forall a, b \in R: a \delta(x) b=0,
$$

we obtain $\delta(x) R \subseteq \operatorname{ann}_{r}(R)$. The $\delta$-primeness implies that $\operatorname{ann}_{r}(R)=\{0\}$. Thus $\delta(x) \in \operatorname{ann}_{\ell}(R)$. But ann $\ell(R)=\{0\}$ too. We have therefore proved that $\delta\left(R^{2}\right)=\{0\}$. Finally, pick an arbitrary $a \in R$ and an arbitrary $x \in R^{2}$. Then

$$
0=\delta(a x)=\delta(a) x+a \delta(x)=\delta(a) x .
$$

It follows that $\delta(R) \subseteq \operatorname{ann}_{\ell}\left(R^{2}\right)$. Using the $\delta$-primeness again, we get $R^{2} \neq\{0\}$ and hence $\operatorname{ann}_{\ell}\left(R^{2}\right)=\{0\}$. We have therefore $\delta=0$.

Let us turn to property $(i i)$. We will proceed by contraposition. Suppose that $\mathrm{C}_{R}(\delta(R)) \neq \mathrm{Z}(R)$. In other words, there is an element $u \in R \backslash \mathrm{Z}(R)$ such that $[u, \delta(a)]=0$ for any $a \in R$. Define $C=\mathrm{C}_{R}\left(\left\{\delta^{m}(u): m \in \mathbb{N}\right\}\right)$. An easy induction shows that

$$
\forall m \in \mathbb{N} \forall a \in R:\left[\delta^{m}(u), \delta(a)\right]=0 .
$$

Hence $\delta(R) \subseteq C$. Moreover, if $x \in R, c \in C$ and $m \in \mathbb{N}$, then

$$
\begin{aligned}
0 & =\left[\delta^{m}(u), \delta(c x)\right]=\left[\delta^{m}(u), \delta(c) x+c \delta(x)\right]=\left[\delta^{m}(u), \delta(c) x\right]+\left[\delta^{m}(u), c \delta(x)\right]= \\
& =\left[\delta^{m}(u), \delta(c)\right] x+\delta(c)\left[\delta^{m}(u), x\right]+\left[\delta^{m}(u), c\right] \delta(x)+c\left[\delta^{m}(u), \delta(x)\right]= \\
& =\delta(c)\left[\delta^{m}(u), x\right] .
\end{aligned}
$$

This yields that $\delta(C) \subseteq A_{\delta}^{0}(u)$. Since $u \notin \mathrm{Z}(R)$, it follows from Lemma 3 that $A_{\delta}^{0}(u)=\{0\}$. Consequently, $\delta(C)=\{0\}$. We therefore obtain

$$
\delta^{2}\left(R^{2}\right) \subseteq \delta^{2}(R)=\delta(\delta(R)) \subseteq \delta(C)=\{0\}
$$

(because $\delta(R) \subseteq C$ ). Thus by property $(i)$, we have $\delta=0$.

\section{THE MAIN THEOREM}

Our main result is a generalization of Herstein's [7, Theorem 2]. 
Theorem 2. Let $R$ be a $\delta$-prime ring. Suppose that

$$
\forall x, y \in R: \delta(x) \delta(y)=\delta(y) \delta(x) .
$$

Then $R$ is commutative whenever one of the following conditions is satisfied:

(1) $\delta^{3} \neq 0$,

(2) $\delta \neq 0$ and $R$ is 2-torsion free.

Proof. Assume that condition (1) is satisfied. Let $S$ be the subring of the ring $R$ generated by $\delta(R)$. Proposition 1 guarantees that there exists a nonzero two-sided $\delta$ ideal $I$ of the ring $R$ contained in $S$. Since the elements of $\delta(R)$ commute pairwise, the subring $S$ is commutative. Therefore so is $I$. The commutativity of the ring $R$ now follows from Lemma 2.

Assume that condition (2) is satisfied. Since the elements of $\delta(R)$ commute pairwise, we have $\delta(R) \subseteq \mathrm{C}_{R}(\delta(R)$ ). Consequently, Proposition 2 ( $i$ i ) yields $\delta(R) \subseteq$ $\mathrm{Z}(R)$. Notice that $E=\{x \in R: \delta(x) \neq 0\}$ is a nonempty set and pick any $x \in E$. Let $y \in R$. Since $\delta(y) \in \mathrm{Z}(R)$ and $\delta(x y) \in \mathrm{Z}(R)$, we get

$$
\begin{aligned}
\delta(x) y x+x^{2} \delta(y) & =\delta(x) y x+x \delta(y) x=\delta(x y) x=x \delta(x y)= \\
& =x \delta(x) y+x^{2} \delta(y) .
\end{aligned}
$$

It follows that $\delta(x) y x=x \delta(x) y$. Hence

$$
\delta(x)[x, y]=\delta(x) x y-\delta(x) y x=x \delta(x) y-x \delta(x) y=0 .
$$

An easy inductive argument now shows that

$$
\text { (*) } \forall n \in \mathbb{N} \backslash\{0\} \forall y \in R: \delta^{n}(x)[x, y]=0 .
$$

Consider the two-sided $\delta$-ideal $J$ of the ring $R$ generated by $\delta(x)$. Notice that $J \neq$ $\{0\}$. Since $\delta^{n}(x) \in \mathrm{Z}(R)$ for all $n \in \mathbb{N} \backslash\{0\}$, the ideal $J$ coincides with the totality of finite sums of elements of the set $A \cup B$, where

$$
A=\left\{a \delta^{n}(x): a \in R, n \in \mathbb{N} \backslash\{0\}\right\}, \quad B=\left\{k \delta^{n}(x): k \in \mathbb{Z}, n \in \mathbb{N} \backslash\{0\}\right\}
$$

(cf. [9, Proposition 2.6]). Property (*) therefore yields $\{[x, y]: y \in R\} \subseteq \operatorname{ann}_{r}(J)$. It follows now from the $\delta$-primeness of $R$ that $[x, y]=0$ for all $y \in R$. Hence we have proved that

$$
\text { (**) } \forall x \in R: \delta(x) \neq 0 \Longrightarrow x \in \mathrm{Z}(R) .
$$

Finally, let $z \in R$ be such that $\delta(z)=0$. Pick some $x_{0} \in E$. Then $\delta\left(z+x_{0}\right) \neq 0$. By property $(* *)$, we therefore get $z+x_{0} \in \mathrm{Z}(R)$. Since $x_{0}$ also belongs to $\mathrm{Z}(R)$, we obtain that $z \in \mathrm{Z}(R)$. The commutativity of the ring $R$ follows. 


\section{REFERENCES}

[1] S. Ali and S. Huang, "On derivations in semiprime rings." Algebr. Represent. Theory, vol. 15, no. 6, pp. 1023-1033, 2012, doi: 10.1007/s10468-011-9271-9.

[2] O. D. Artemovych and M. P. Lukashenko, "Lie and Jordan structures of differentially semiprime rings." Algebra Discrete Math., vol. 20, no. 1, pp. 13-31, 2015.

[3] M. Ashraf, S. Ali, and C. Haetinger, "On derivations in rings and their applications." Aligarh Bull. Math., vol. 25, no. 2, pp. 79-107, 2006.

[4] M. N. Daif, "Commutativity results for semiprime rings with derivations." Int. J. Math. Math. Sci., vol. 21, no. 3, pp. 471-474, 1998, doi: 10.1155/S0161171298000660.

[5] N. A. Dar and S. Ali, "On *-commuting mappings and derivations in rings with involution." Turk. J. Math., vol. 40, no. 4, pp. 884-894, 2016, doi: 10.3906/mat-1508-61.

[6] I. N. Herstein, Rings with involution. Chicago-London: The University of Chicago Press, 1976.

[7] I. N. Herstein, "A note on derivations." Can. Math. Bull., vol. 21, pp. 369-370, 1978, doi: 10.4153/CMB-1978-065-X.

[8] C. R. Jordan and D. A. Jordan, "Lie rings of derivations of associative rings." J. Lond. Math. Soc., II. Ser., vol. 17, pp. 33-41, 1978, doi: 10.1112/j1ms/s2-17.1.33.

[9] K. Kular, " On basic properties of $\delta$-prime and $\delta$-semiprime rings." Technical Transactions. Fundamental Sciences (Czasopismo Techniczne. Nauki Podstawowe), vol. 1-NP, pp. 107-119, 2016, doi: 10.4467/2353737XCT.16.144.5755.

[10] J. Pinter-Lucke, “Commutativity conditions for rings: 1950-2005.” Expo. Math., vol. 25, no. 2, pp. 165-174, 2007, doi: 10.1016/j.exmath.2006.07.001.

[11] M. S. Tammam El-Sayiad and N. O. Alshehri, "On an extension of a result of Herstein." J. Algebra Appl., vol. 12, no. 8, p. 10, 2013, doi: 10.1142/S0219498813500515.

Author's address

Kamil Kular

Cracow University of Technology, Institute of Mathematics, ul. Warszawska 24, 31-155 Kraków, Poland

E-mail address: kkularepk.edu.pl 